\title{
A medical student's reflection on intercalation: retraction
}

The IJS Publishing Group have agreed to a request from the authors to withdraw their education article, "A medical student's reflection on intercalation" ${ }^{[1]}$ from publication in IJS Oncology. An error on the IJS Publishing Group site meant the authors expectations for indexing were not met. The work is not flawed.

\section{Reference}

[1] Janmohamed IK. A medical student's reflection on intercalation. IJS Oncology 2019;4:e75. 